\title{
A review of the safety and clinical utility of contrast echocardiography
}

Siang Chew $\underline{C h a i}^{1}$, MBBS, MRCP, Puay Joo $\underline{T a n}^{1}$, RDCs, Khim Leng Tong ${ }^{1}$, MBBS, FRCP

\begin{abstract}
There are limitations to the sensitivity and specificity of conventional two-dimensional echocardiograms in making an accurate diagnosis in certain patient populations. This led to the development of specific contrast-enhancing agents with the following characteristics: small enough to cross the pulmonary capillary bed, remain stable throughout the length of the procedure, do not dissolve in blood, and rapidly cleared from the body with low toxicity. Unfortunately, the use of contrast echocardiography has not taken off as expected. The low take-up rate among clinicians can largely be attributed to the black box warning by the United States Food and Drug Administration in 2007, after the coincidental occurrence of four patient deaths and about 190 severe cardiopulmonary reactions shortly after contrast agent administration. In this article, we address the clinical safety of contrast agents, share our institution's experience in using it and elaborate on the clinical indications of contrast echocardiography.
\end{abstract}

Keywords: clinical safety, clinical utility, contrast echocardiography

\section{INTRODUCTION}

Contrast echocardiography is not widely ordered as a diagnostic imaging modality, probably due to poor understanding, even among cardiologists, of its clinical application and safety profile. This article serves to demystify and clarify conditions in which adding a contrast agent to routine echocardiography can result in a very powerful diagnostic tool. Contrast echocardiography is simply an imaging technique for enhancing the endocardium and blood border wall. Agitated saline microbubbles were previously used to examine the right heart (mainly the right atrium) and identify intracardiac shunts. These air microbubbles are short-lasting and could not even reach the left heart, as they would have diffused into the lungs while passing through the pulmonary circulation.

\section{ULTRASOUND ENHANCING AGENTS}

In order to evaluate the left heart chambers, the contrast agent must be small and stable so as to remain intact after crossing the pulmonary circulation to reach the left heart after being administered intravenously. The use of ultrasound enhancing agents (UEAs) consisting of inert gases (perfluorocarbons and sulfur hexafluoride), which have lower solubility and diffusivity than air, encapsulated with lipid or protein shells markedly increases the lifespan of the contrast agent after injection. These modifications have resulted in more stable microbubbles $(<6 \mu \mathrm{m})$ that can traverse the pulmonary or systemic microcirculations without getting lysed. ${ }^{(1)}$ Table I shows the commons UEAs that have been clinically approved for use. We have experience using both Definity and SonoVue in our institution.

\section{Safety for use}

The United States Food and Drug Administration (FDA) approved two UEAs, Optison and Definity, for left ventricular (LV) endocardial border delineation after Phase III multicentre trials in 2001. ${ }^{(2,3)}$ In October 2007, the FDA issued revised labelling requirements, including a black box warning for these UEAs. This happened after the coincidental occurrence of four patient deaths and about 190 severe cardiopulmonary reactions shortly after UEA administration (Definity or Optison).

A retrospective study in 2009 by Dolan et al analysed the usage of UEAs in 18,749 patients who had suboptimal baseline images and subsequently underwent contrast stress echocardiography. The authors concluded that UEAs are safe to use in patients suspected of having coronary artery disease. ${ }^{(4)}$ In a prospective study published in 2012, Weiss et al evaluated the clinical safety of Definity and aimed to assess the risk of adverse cardiopulmonary events occurring during or within 30 minutes after administering Definity. They demonstrated that Definity was well tolerated in routine clinical practice in patients with a high prevalence of cardiopulmonary disease. ${ }^{(5)}$ On a similar note, Main et al reported in 2013 that at a clinically relevant dose of $0.5 \mathrm{~mL}$ Optison, pulmonary artery systolic pressure or pulmonary vascular resistance did not change after intravenous administration. ${ }^{(6)}$ These two studies reinforced the safety of UEAs for cardiac indications.

Since then, numerous studies have demonstrated the safety of UEAs in various clinical settings (e.g. emergency and intensive care departments) as well as in the paediatric population. UEAs are also safe to use in patients undergoing stress echocardiography and in those suspected of having pulmonary hypertension and intracardiac shunts. In 2008, the FDA downgraded the contraindications listed in the black box to warnings. In 2011, it removed the mandatory 30 minutes of monitoring after UEA administration in patients with pulmonary hypertension or unstable cardiopulmonary conditions. In late 2016 and early 2017, the FDA further removed the contraindication for

${ }^{1}$ Department of Cardiology, Changi General Hospital, Singapore

Correspondence: Dr Chai Siang Chew, Consultant, Department of Cardiology, Changi General Hospital, 2 Simei Street 3, Singapore 529889. chai.siang.chew@singhealth.com.sg 
Table I. Ultrasound contrast agents that are clinically approved.

\begin{tabular}{|lllll|}
\hline Name & Date approved & Shell material & Gas & Application \\
\hline Optison & 1986 & Cross-linked serum albumin & Octafluoropropane & LVO \\
\hline SonoVue & 2011 & Phospholipid & Sulfur hexafluoride & LVO, microvascular enhancement (liver and breast) \\
\hline Definity & 2011 & Phospholipid & Octafluoropropane & Echocardiography, liver/kidney imaging \\
\hline
\end{tabular}

LVO: left ventricular opacification

administration of Optison, Lumason/SonoVue and Definity in patients with intracardiac shunts. In fact, the American Society of Echocardiography (ASE) 2018 guideline recommends routine use of contrast agents in evaluating patients with patent foramen ovale and small right-to-left shunts. ${ }^{(7,8)}$

Contrast agents can also be used in LV assist devices and extracorporeal membrane oxygenators (ECMOs), although care must be taken to avoid ECMO circuit shutdowns due to bubble-sensing safety systems. ${ }^{(9)}$ As of August 2017, the only contraindication was intra-arterial injection; and for SonoVue and Definity, known hypersensitivity to sulfur hexafluoride and perflutren, respectively. In summary, despite initial FDA contraindications to the contrary, there is substantial evidence to show that echocardiographic contrast agents are not only safe in critically ill patients but also that important diagnostic data can potentially be derived from these studies.

\section{THE CHANGI GENERAL HOSPITAL EXPERIENCE}

At Changi General Hospital (CGH), we started using Definity in 2008 and later switched to SonoVue in 2012 due to a cost issue. In 2012-2017, there were a total of 395 contrast echocardiographic studies performed with SonoVue, including both transthoracic and stress echocardiograms. For Definity, there were two cases of non-specific back pain. In the one case of anaphylactic shock with SonoVue, the patient responded promptly to fluid resuscitation with no further clinical sequel. Currently, we use UEAs in about $1 \%$ of our stress and regular echocardiography.

\section{CLINICAL UTILITY OF CONTRAST ECHOCARDIOGRAPHY}

The main indication for contrast echocardiography is for assessment of LV ejection fraction and regional wall motion abnormalities (RWMA). ${ }^{(7-12)}$ Left ventricular opacification (LVO) with UEA helps to accurately delineate the endocardial border, especially in suboptimal cases, as defined by inability to visualise at least two out of the 17 myocardial segments. For clinical settings in which accurate serial assessments of the LV ejection fraction are required (e.g. patients undergoing chemotherapy or potentially requiring a cardiovascular implantable electronic device) and when visualisation of the endocardium is critical (e.g. RWMA assessment in stress echocardiography), LVO with UEA should be contemplated.

Contrast can be administered during echocardiography to confirm or exclude certain LV structural abnormalities. (a) The characteristic spade-like appearance of the LV cavity, which is characteristic of apical hypertrophic cardiomyopathy, can be better delineated with UEA. ${ }^{(13)}$ It also allows the echocardiographer to measure the myocardial thickness more accurately with confidence. (b) For LV non-compaction, the non-compacted layer can be differentiated more easily against the compacted myocardium by enhancing the LV cavity with UEA. ${ }^{(14)}$ The ASE recommends a non-compacted to compacted ratio of $>2: 1$ when using contrast to make the diagnosis of LV non-compaction (Fig. 1). (c) Weinsaft et al ${ }^{(15)}$ have demonstrated the superiority of contrast over non-contrast echocardiography for the detection of LV, especially apical thrombi (Fig. 2), with improved sensitivity $(61 \%$ vs. $33 \%, p<0.05)$ and accuracy $(92 \%$ vs. $82 \%, p<0.01)$. As such, the ASE recommends that UEA be used in patients with severely impaired LV systolic function and in whom the apex is not well visualised, to assess for suspicious thrombi. (d) LV apical aneurysm is characterised by dilatation of thin and scarred apical myocardium. The use of UEAs helps to delineate the aneurysm and even uncover concomitant LV apical thrombus, especially when the apex is not well visualised despite using multiplanar imaging technique on non-contrast echocardiography. (e) LV pseudoaneurysm, free wall rupture and ventricular septal defects are life-threatening complications that can develop in patients following myocardial infarction. ${ }^{(16)}$ However, such patients, more often than not, are intubated due to acute pulmonary oedema from cardiogenic shock and, thus, they will have suboptimal echocardiography windows due to anatomy and supine positioning. Contrast echocardiography can delineate abnormal anatomical structures more clearly and document the presence or absence of extracardiac extravasation of contrast agent in cases of suspected ventricular free wall rupture.

\section{COST-EFFECTIVENESS OF ULTRASOUND ENHANCING AGENTS}

Studies ${ }^{(17-20)}$ have shown that contrast echocardiography helps to save healthcare costs through, first, reducing costlier or more invasive testing downstream in patients with an initially non-diagnostic echocardiogram, as contrast echocardiography can clearly demonstrate the diagnosis. In short, contrast echocardiography has higher sensitivity and specificity for certain cardiac conditions and should be applied when clinically indicated. Second, laboratory efficiency improves due to the shorter scanning time of contrast echocardiography in patients with poor echo windows. The echocardiographers can spend less unproductive time searching for optimal echocardiography images.

\section{CONCLUSION}

This article aims to improve practising doctors' knowledge of the indications, risks and benefits of contrast echocardiography. The future potential applications of UEAs in gene and drug delivery are beyond the scope of this article. Contrast echocardiography 

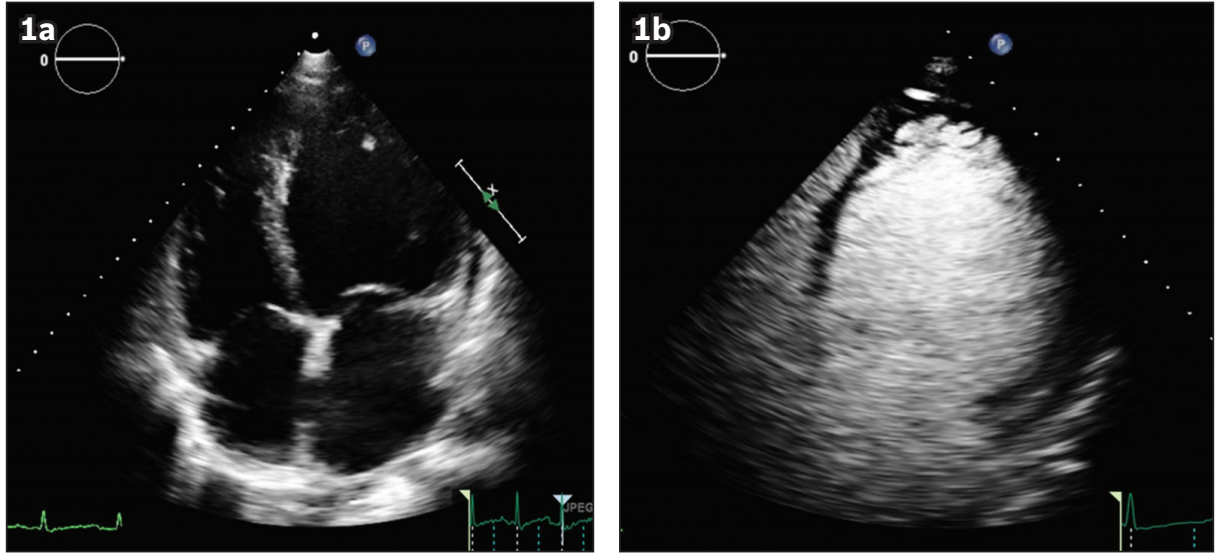

Fig. 1 (a) Pre-contrast and (b) post-contrast US images in apical four-chamber view show the features of left ventricular (LV) non-compaction with SonoVue contrast administration. (b) Numerous, excessively prominent trabeculations and deep intratrabecular recesses are seen. The diagnosis of LV non-compaction was confirmed with cardiac magnetic resonance imaging.
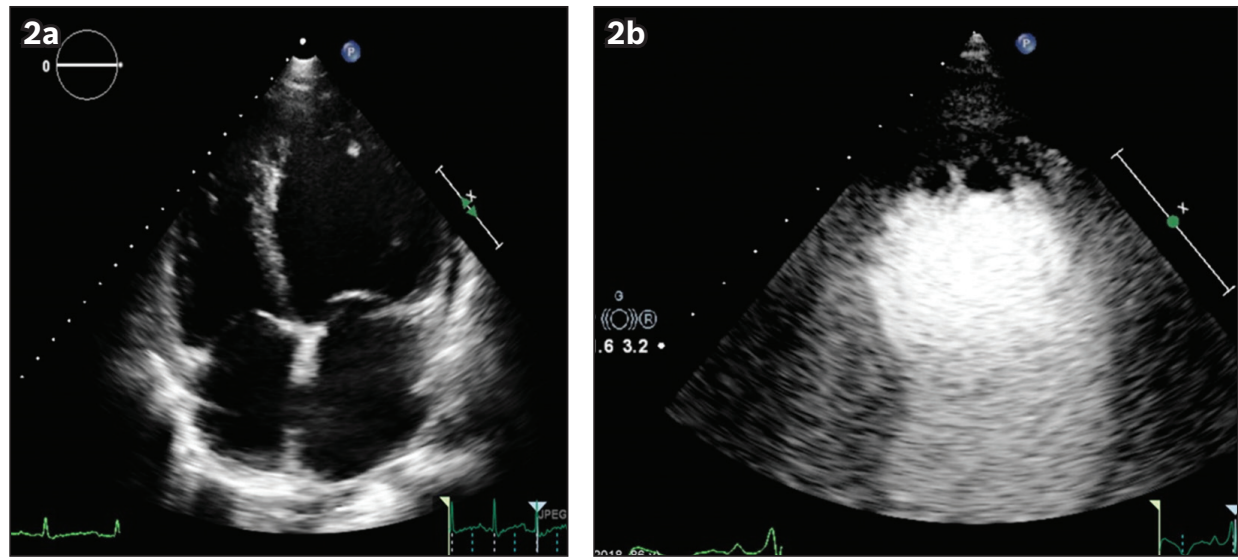

Fig. 2 (a) Pre-contrast and (b) post-contrast images in apical four-chamber view show an improved ability to detect an apical thrombus.

should be considered on a case-by-case basis depending on the clinical indication. As we have demonstrated, it is a powerful diagnostic tool that is readily available to complement conventional two-dimensional echocardiography.

\section{REFERENCES}

1. Meerbaum S. Development and validation of MC-2DE methodology. In: Meerbaum S, Meltzer R, eds. Myocardial Contrast Two-Dimensiona Echocardiography. Boston: Kluwer Academic Publishers, 1989: 7-44.

2. Kitzman DW, Goldman ME, Gilam LD, et al. Efficacy and safety of the nove ultrasound contrast agent perflutren (definity) in patients with suboptimal baseline left ventricular echocardiographic images. Am J Cardiol 2000; 86:669-74.

3. Cohen JL, Cheirif J, Segar DS, et al. Improved left ventricular endocardial border delineation and opacification with OPTISON (FS069), a new echocardiographic contrast agent. Results of a phase III multicenter trial. J Am Coll Cardiol 1998; 32:746-52.

4. Dolan MS, Gala SS, Dodla S, et al. Safety and efficacy of commercially available ultrasound contrast agents for rest and stress echocardiography a multicenter experience. J Am Coll Cardiol 2009; 53:32-8.

5. Weiss RJ, Ahmad M, Villanueva F, et al; CaRES Investigators. CaRES (Contras Echocardiography Registry for Safety Surveillance): a prospective multicenter study to evaluate the safety of the ultrasound contrast agent definity in clinical practice. J Am Soc Echocardiogr 2012; 25:790-5.

6. Main ML, Grayburn PA, Lang RM, et al. Effect of Optison on pulmonary artery systolic pressure and pulmonary vascular resistance. Am J Cardiol 2013; 112:1657-61

7. Porter TR, Abdelmonieum S, Belcik JT, et al. Guidelines for the cardiac sonographer in the performance of contrast echocardiography: a focused update from the American Society of Echocardiography. J Am Soc Echocardiogr 2014 27: 797-810.

8. Porter TR, Mulvagh SL, Abdelmoneim SS, et al. Clinical applications of ultrasonic enhancing agents in echocardiography: 2018 American Society of Echocardiography Guidelines Update. J Am Soc Echocardiogr 2018; 3:241-74.
9. Bennett CE, Tweet MS, Michelena HI, Schears GJ, Mulvagh SL. Safety and feasibility of contrast echocardiography for ECMO evaluation. JACC CardiovasC Imaging 2017; 10:603-4.

10. Mulvagh SL, Rakwski H, Vannan MA, et al; American Society of Echocardiography. American Society of Echocardiography consensus statement on the clinical applications of ultrasonic contrast agents in echocardiography. J Am Soc Echocardior 2008; 21:1179-201.

11. Kurt M, Shaikh KA, Peterson L, et al. Impact of contrast echocardiography on evaluation of ventricular function and clinical management in a large prospective cohort. J Am Coll Cardiol 2009; 53:802-10.

12. Bhatia VK, Senior R. Contrast echocardiography: evidence for clinical use. J Am Soc Echocardiogr 2008; 21:409-16.

13. Thanigaraj S, Pérez JE. Apical hypertrophic cardiomyopathy: echocardiographic diagnosis with the use of intravenous contrast image enhancement. J Am Soc Echocardiogr 2000; 13:146-9.

14. Chow CM, Lim KD, Wu L, Leong-Poi H. Images in cardiovascular medicine. Isolated left ventricular noncompaction enhanced by echocontrast agent. Circulation 2007; 116:e90-1.

15. Weinsaft JW, Kim RJ, Ross M, et al. Contrast-enhanced anatomic imaging as compared to contrast-enhanced tissue characterization for detection of left ventricular thrombus. JACC Cardiovasc Imaging 2009; 8:969-79.

16. Moreno R, Zamorano JL, Almería C, et al. Usefulness of contrast agents in the diagnosis of left ventricular pseudoaneurysm after acute myocardial infarction. Eur J Echocardiogr 2002; 3:111-6.

17. Thanigaraj S, Nease RF Jr, Schechtman KB, et al. Use of contrast for image enhancement during stress echocardiography is cost-effective and reduces additional diagnostic testing. Am J Cardiol 2001; 87:1430-2.

18. Castello R, Bella JN, Rovner A, et al. Efficacy and time-efficiency of a "sonographer-driven" contrast echocardiography protocol in a high-volume echocardiography laboratory. Am Heart J 2003; 145:535-41.

19. Vlassak I, Rubin DN, Odabashian JA, et al. Contrast and harmonic imaging improves accuracy and efficiency of novice readers for dobutamine stress echocardiography. Echocardiography 2002; 19:483-8.

20. Yong Y, Wu D, Fernandes $V$, et al. Diagnostic accuracy and cost-effectiveness of contrast echocardiography on evaluation of cardiac function in technically very difficult patients in the intensive care unit. Am J Cardiol 2002; 89:711-8. 